EchoGéo

ECHOGEO- $5 \mid 2008$

"Discipliner" la sexualité

\title{
Acquisitions de la Bibliothèque Géographie
}

\section{Bernadette Joseph}

\section{OpenEdition}

Journals

Édition électronique

URL : https://journals.openedition.org/echogeo/4514

DOI : $10.4000 /$ echogeo.4514

ISSN : 1963-1197

\section{Éditeur}

Pôle de recherche pour l'organisation et la diffusion de l'information géographique (CNRS UMR 8586)

\section{Référence électronique}

Bernadette Joseph, « Acquisitions de la Bibliothèque Géographie », EchoGéo [En ligne], 5 | 2008, mis en ligne le 30 mai 2008, consulté le 02 août 2021. URL : http://journals.openedition.org/echogeo/4514 ; DOI : https://doi.org/10.4000/echogeo.4514

Ce document a été généré automatiquement le 2 août 2021

EchoGéo est mis à disposition selon les termes de la licence Creative Commons Attribution - Pas d'Utilisation Commerciale - Pas de Modification 4.0 International (CC BY-NC-ND) 


\title{
Acquisitions de la Bibliothèque Géographie
}

\author{
Bernadette Joseph
}

\section{BIBLIOGRAPHIE}

\section{Afrique}

BEN YAHMED, Atlas de Djibouti, Paris, Les éd. J.A., cop. 2007, G 19-3325

BERNARDIE-TAHIR, L'autre Zanzibar : géographie d'une contre-insularité, Paris ; Pessac ; Limoges, Karthala : ADES-Dymset : GEOLAB-Limoges, impr. 2008, G 19-3370

BRÉDELOUP, Sylvie, La Diams'pora du fleuve Sénégal : sociologie des migrations africaines, Toulouse ; Paris, Presses Universitaires du Mirail : IRD éditions, impr. 2007,

G 19-3347

BRIAND, Virginie, Manger au quotidien : la vulnérabilité des familles urbaines en Afrique, Paris, IRD : Karthala, cop. 2007, G 19-3368

CAZES-DUVAT, Virginie, L lie de Birdaux Seychelles : un exemple de développement durable?, Paris ; Budapest ; Torino, l'Harmattan, 2004, G 19-3114

CHABOUD, FROGER, MÉRAL, Madagascar face aux enjeux du développement durable : des politiques environnementales à l'action collective locale, Paris, Éd. Karthala, DL 2007, G 19-3348

COLIN, Loïc, GENTIL, PAPAZIAN, L'Office du Niger : du travailleur forcé... au paysan syndiqué, Paris ; Institut de recherches et d'applications des méthodes de développement [prod.], 2006, DVD 75 
DIDACE-AMBOULOU, Hygin, Administration du Territoire et les Collectivités locales en République du Congo, l'Harmattan, 2008

ESSOMBE EDIMO NYA BONABEBEY, Jean-Roger, Spatialité et développement économique à Douala : entre le hasard et la nécessité, Paris, l'Harmattan, DL 2007, G 19-3364

FALL, Abdou Salam, DE SWAAN, Bricoler pour survivre : perceptions de la pauvreté dans l'agglomération urbaine de Dakar, Paris, Khartala, impr. 2007, G 19-3363

GAFSI, DUGUÉ, JAMIN, BROSSIER, Exploitations agricoles familiales en Afrique de l'Ouest et du Centre, Versailles, Éditions Quae, 2007, G 19-3365

HAFERBURG, Christoph, Umbruch oder Persistenz ? : sozialräumliche Differenzierungen in Kapstadt, Hamburg, Institut für Geographie der Universität Hamburg, 2007, G 19-3357

JAUZE, L'île Maurice face à ses nouveaux défis, Paris ; Saint-Denis (Réunion), l'Harmattan : Université de la Réunion, DL 2008, G 19-3378

KIALO, Paulin, KALAORA, Anthropologie de la forêt : populations pové et exploitants forestiers français au Gabon, Paris, L'Harmattan, 2007, G 19-3355

LAPARRA, Maurice, Alucam un Destin africain, Mirabeau (Vaucluse), REF 2 C, 2008

OUÉDRAOGO, PICHÉ, Dynamique migratoire, insertion urbaine et environnement au Burkina Faso : au-delà de la houe, Paris, l'Harmattan, DL 2007, G 19-3366

Rapport sur le développement en Afrique, Paris, Economica, [19..]-, G 19-2823

RAYNAUT, Claude, Recherches multidisciplinaires sur la région de Maradi : rapport de synthèse, Paris ; Bordeaux, DGRST : Université de Bordeaux 3, 1980, G 19-3362

SANDRON, Frédéric, Populations et développement dans les Hautes Terres de Madagascar, Paris, L'Harmattan, 2007, G 19-3369

TANTCHOU YAKAM, Josiane Carine, Epidémie et politique en Afrique : maladie du sommeil et tuberculose au Cameroun, Paris, l'Harmattan, DL 2007, G 19-3361

VAILLANT, Zoé, CYRULNIK, La Réunion, koman i lé? : territoires, santé, société, Paris, Presses Universitaires de France, 2008, G 19-3359

\section{Amérique du nord}

DEGE, Wilhelm, Die Westküste Grönlands : Bevölkerung, Wirtschaft und Siedlung im Strukturwandel, Bremen, [s.n.], 1965, G 20-1601

FOSTER, LECLAIR, Contemporary issues in mental health : concepts, policy, andpractice, Victoria, B.C., Western Geographical Press, cop. 2007, G 20-1606

LEFÈVRE, Christian, Les villes des Etats-Unis, Paris ; Milan ; Barcelone ; Mexico, Masson, 1988,

G 20-1259

PREWITT, Kenneth, Politics and science in census taking, New York ; Washington, DC, Russell Sage Foundation : Population Reference Bureau, 2003, G 20-1604 


\section{Amérique Latine}

Desarrollo sostenible y medio ambiente en Repûblica Dominicana : medios naturales, manejo histôrico, conservaciônyprotecciôn, Sevilla, Consejo Superior de Investigaciones Cientificas : Universidad de Sevilla, impr. 2005, G 21-1646

FALCADE, MANDELLI, FLORES, FASOLO, POTTER, Vale dos vinhedos : caracterizaçào geogrâfica da regiâo, Caxias do Sul ; Bento Gonçalves, USC, Universidade de Caxias do Sul : Embrapa, Empresa brasileira de pesquisa agropecuâria, 1999, G 21-1645

G 21-1635

LAMBOURDIÈRE, Éric, Les Caraïbes dans la géopolitique mondiale, Paris, Ellipses, impr. 2007, G 21-1631

MAM-LAM-FOUCK, Comprendre la Guyane d'aujourd'hui : un département français dans la région des Guyanes, Matoury (Guyane), Ibis rouge éditions, 2007, G 21-1626

MAM-LAM-FOUCK, Comprendre la Guyane d'aujourd'hui : un département français dans la région des Guyanes, Matoury (Guyane), Ibis rouge éditions, 2007, G 21-1626

MAVOUNGO, Joseph, SAFFACHE, Le séisme majeur du 11 janvier 1839 de la Martinique : élément de mémoire collective, d'éducation et de prévention du risque sismique aux Antilles françaises, Paris, Publibook, 2008, G 21-1647

Naturschutz und Schutzgebiete aufKuba, Miinchen, Départaient fur Géographie der Univ. Munchen, 2007, G 21-1648

PELLEGATTI, Empreendedorismo consciente na Amazônia, São Paulo, TV1 editorial, 2007, cop. 2007, G 21-1640

Política ambiental integrada para o desenvolvimento sustentável : relatorio de gestão 2003-2006, Brasília, Ministério do Meio ambiente, 2007, G 21-1636

PUGH, MOMSEN, Environmentalplanning in the Caribbean, Hampshire, England ; Burlington, USA, Ashgate, 2006, G 21-1649

REVET, Sandrine, Anthropologie d'une catastrophe : les coulées de boue de 1999 au Venezuela, Paris, Presses Sorbonne nouvelle, DL 2007, G 21-1634

THÉRY, Hervé, Le Brésil, Paris, A. Colin, 2000, G 21-1193

VERNER, EGSET, Social resilience and state fragility in Haiti, Washington, World Bank.

\section{Asie}

BOZDEMIR, Mevlut, Turquie entre islam et Europe, Paris, Ellipses, impr. 2007, G 17-2601

GUERMOND, Yves, La Chine, Paris, Belin, DL 2007, G 17-2567

LANDY, Frédéric, L'Inde ou le grand écart, Paris, la Documentation française, DL 2007, G 17-2611

THIBAULT, Christel, BRUNEL, L'archipel des camps : l'exemple cambodgien, Paris, Presses universitaires de France, DL 2008, G 17-2612 


\section{Atlas}

Atlas de la coopération transfrontalière : dynamiques transfrontalières et projets de territoires, Paris, Mission opérationnelle transfrontalière, 2007, AQ 13-122

Atlas de la República Argentina, Buenos Aires, J. Ruland, 1898, AF 21-39

FOSTER, Leslie Thomas, The British Columbia atlas ofwellness, Victoria, B.C., Western

Geographical Press, cop. 2007, AQ 20-25

Geográfia de Centroamérica y del Caribe, Tegucigalpa, Ed. Ramses, 2006, AQ 21-41

LITALIEN, Raymonde, La mesure d'un continent : atlas historique de l'Amérique du Nord, 1492-1814, Sillery ; Paris, Septentrion : Presses de l'Université Paris-Sorbonne, impr. 2007, AQ 20-24

\section{Congrès}

ANSART, Pierre, Longévité et politiques publiques : Approches comparées France-Japon, Nancy, Presses universitaires de Nancy, 2007, G 3-2929

ANTOINE, MARGUERIE, Bocages et sociétés : actes du colloque organisé à l'université de Rennes 2, 29, 30 septembre et $1^{\text {er }}$ octobre 2004, Rennes, Presses universitaires de Rennes, 2007, G 3-2923

ANTOINE, MARGUERIE, Bocages et sociétés : actes du colloque, Rennes, Presses universitaires de Rennes, 2007, G 3-2923

BODY-GENDROT, SPIERENBURG, Violence in Europe : historical and contemporary perspectives, New York, Springer, cop. 2008, G 3-2919

BOESEN, MARFAING, Les nouveaux urbains dans l'espace Sahara-Sahel : un cosmopolitisme par le bas, Paris ; Berlin, Karthala : Zentrum moderner Orient, c2007, G 3-2930

CHEMLA, BILLOT, Colombo : environmental issues at stake, Colombo, CMC ed., impr. 2007, G 3-2920 DOUÉTIL, CAILLEAUX, Des rivières, des hommes, une longue histoire : actes du colloque du SIARCE, Corbeil-Essonnes, 4-5 novembre 2005, Paris, AEDEH, 2007, G 3-2936

FERNANDES, MARQUES, SUZUKI, Geografia agrária : teoria e poder, São Paulo, Expressão popular, 2007, G 3-2926

FONSECA, CADEDDU, GALLINARI, In viaggio verso le Americhe, Italiani e Portoghesi in Brasile : Convegno di studi per il V centenario della scoperta del Brasile, Cagliari, 30 novembre-2 dicembre 2000, Roma, Società geografica italiana, 2004, G 3-2937

FRUSTIER, Les identités insulaires face au tourisme : actes du colloque, La Roche-sur-Yon, Éd. Siloë, impr. 2007, G 3-2935

FRUSTIER, Les identités insulaires face au tourisme : actes du colloque, La Roche-sur-Yon, Éd. Siloë, impr. 2007, G 3-2935

Infrastrutture e competitività : quale scénario per il sistema Italia : Roma, 7 giugno 2006, Roma, Unioncamere, impr. 2006, G 3-2957

KOFF, Deceiving (dis)appearances : analyzing current developments in European and North American border regions, Bruxelles, P.I.E. Peter Lang, cop. 2007, G 3-2921 
LEROUSSEAU, La décentralisation à la croisée des chemins : actes du colloque du 16 février 2007, Paris, L'Harmattan, DL 2007, G 3-2931

Les paysages à l'époque moderne, [Paris], PUPS, Presses de l'Université Paris-Sorbonne, DL 2007, G 3-2932

LOCOH, NGUESSAN, MAKINWA-ADEBUSOYE, Genre et sociétés en Afrique : implications pour le développement, Paris, Institut national d'études démographiques, DL 2007, G 3-2924

MARTENS, Identité et question régionale en Europe, Villeneuve d'Ascq, Presses universitaires du Septentrion diffusion, DL 2007, G 3-2933

PONDI, Immigration et diaspora : un regard africain, Paris, Maisonnneuve \& Larose-Afredit, 2007, G 3-2927

Territorio, ciudad y educación : actas, Barcelona, APEC, 2007, G 3-2934

TREMOLIÈRES, Michèle, Protéger, restaurer et gérer les zones alluviales : pourquoi et comment : why and how ?, Paris, Ed. Tec. \& doc. : Lavoisier, DL 2007, G 3-2952

\section{Dictionnaires, répertoires}

JOLY, Répertoire des géographes français 2007, Paris, CNRS-UMR-PRODIG, 2007, G 1-759

LÉVY, LUSSAULT, Dictionnaire de la géographie, Paris, Belin, DL 2003, W 289

\section{Europe}

AGNOLETTI, Mauro, Ilparco delpaesaggio rurale appenninico di Moscheta, Pisa, Pacini, cop. 2007, G 15-5756

ALEXANDRE-COLLIER, HELLENCOURT, SCHNAPPER, Le Royaume-Uni et l'Union européenne depuis 1997, Dijon, Éditions universitaires de Dijon, 2007, G 15-5728

AMORUSO, Onofrio, MAUTONE, Parco nazionale del Pollino, Roma, Ministero dell'ambiente e della tutela del territorio : Società geografica italiana, 2006, G 15-5743

B ARJOT, Penser et construire l Europe : l'idée et la construction européenne de Versailles à Maastricht, 1919-1992, [Paris], CNED : Éd. Sedes, impr. 2007, G 15-5758

BERLAN-DARQUÉ, LUGINBUHL, TERRASSON, Paysages : de la connaissance à l'action, Versailles, Ed. Quae, DL 2007, G 15-5721

BOCQUET, Denis, Rome ville technique : 1870-1925 : une modernisation conflictuelle de l'espace urbain, Rome, École française de Rome, 2007, G 15-5720

BOCQUET, Denis, Rome ville technique : 1870-1925 : une modernisation conflictuelle de l'espace urbain, Rome, Ecole française de Rome, 2007, G 15-5720

BOULMIER, CHAS SAIGNE, Ville et insécurité en Europe occidentale, $\mathrm{XIX}^{\mathrm{e}} \mathrm{XXI}^{\mathrm{e}}$ siècles, Paris, la Documentation française, DL 2007, G 15-5768

BUZZETTI, MONTANARI, Nuovi scenari turistici per le aree montane : Abruzzo e Trentino, sviluppo locale e competitività del territorio, Trento, V. Trentini, impr. 2006, G 15-5718

BYRON, Margaret, Migration in comparative perspective : Caribbean communities in Britain and France, New York, Routledge, 2008, G 15-5730 
CECCHINI, MACIOCCO, Al centro le periferie : il ruolo degli spazi pubblici e dell'attivazione delle energie sociali in un'esperienza didattica per la riqualificazione urbana, Milano, F. Angeli, cop. 2007, G 15-5695

CHEVALIER, PERCEBOIS, CHALMIN, COHEN, Gaz et électricité : un défi pour l'Europe et pour la France, Paris, La documentation française, DL 2008, G 15-5767

CHRISTALLER, Walter, BASKIN, Central places in Southern Germany, Englewood Cliffs (New Jersey), Prentice-Hall, [cop. 1966], G 15-1357

CIATTONI, La Russie, Paris, Éd. Sedes, impr. 2007, G 15-5660

D'APONTE, Tullio, Parco nazionale del Cilento e Vallo di Diano, Roma, Ministero dell'ambiente e della tutela del territorio : Società geografica italiana, 2006, G 15-5742

DELWIT, REA, SWYNGEDOUW, Bruxelles ville ouverte : immigration et diversité culturelle au coeur de l'Europe, Paris, l'Harmattan, DL 2008, cop. 2007, G 15-5731

e della tutela del territorio : Società geografica italiana, 2006, G 15-5744

FACCIOLI, Marina, MAUTONE, Parco nazionale della Sila, Roma, Ministero dell'ambiente

FEDERAL STATE STATISTICS SERVICE, Russia in figures 2006 : statistical handbook, Moscow, Rosstat, 2006, G 15-5719

FLETCHER, George, The Provinces of Ireland, Cambridge, University Press, 1921-1922, G 15-5771

FLÖSS, I nomi locali della Val di Ledro, Trento, Provincia Autonoma di Trento, Soprintendenza per i Beni librari e archivistici, 2006, G 15-5732

FRAGOLA, Fleur, CAUTRÈS, Vers une politique ferroviaire européenne ? : l'Europe à toute vapeur ?, Paris, L'Harmattan, DL 2007, G 15-5727

G 15-5726

GADDONI, Italia regione d'Europa, Bologna, Pàtron ed., 2007, G 15-5738

GENTILESCHI, Geografie dell'immigrazione : stranieri in Sardegna, Bologna, Pàtron ed., 2007, G 15-5736

HAËNTJENS, Jean, Le pouvoir des villes ou l'art de rendre désirable le développement durable, La Tour-d'Aigues (Vaucluse), Éd. de l'Aube, impr. 2008, G 15-5749

HALLAIR, Gaëlle Frédérique, ROBIC, Le géographe Emmanuel de Martonne et l'Europe centrale, Paris, Pôle de recherche pour l'organisation et la diffusion de l'information géographique, 2007, G 15-5748

HUETZ DE LEMPS, Alain, L'économie de l'Espagne, Paris ; Milan ; Barcelone ; Mexico, Masson, 1989, A 15.321

La Russie et l'Europe, Paris, Choiseul, 2007, G 15-5764

LACONTE, KALLAS, PICQUÉ, Bruxelles, la Belgique et l'Europe : un urbanisme cosmopolite, Lyon, CERTU, Impr.2007, G 15-5722

LOYAT, Jacques, La politique agricole commune : une politique en mutation, Paris, la Documentation française, impr. 2008, G 15-5766

MAUREL, LACQUEMENT, Agriculture et ruralité en Europe centrale, Montreuil, Aux lieux d'être :

CEFRES, impr. 2007, G 15-5694 
MAUTONE, Maria, Parco nazionale del Vesuvio, Roma, Ministero dell'ambiente e della tutela del territorio : Società geografica italiana, 2006, G 15-5741

MÉRAND, LAVENEX, Sécurité extérieure de l'UE : nouveaux territoires, nouveaux enjeux, Paris, l'Harmattan, DL 2007, G 15-5729

MESPOULET, Martine, PORTER, Construire le socialisme par les chiffres : enquêtes et recensements en URSS de 1917 à 1991, Paris, Institut national d'études démographiques, DL 2008, G 15-5765

MORANDI, Corinna, Milan : the great urban transformation, Venezia, Marsilio, 2007, G 15-5769

MUNAFÔ, Sébastien, PINI, PIGUET, Autoroute, mobilité et habitat : étude des effets territoriaux du tronçon autoroutier de la Vue-des-Alpes : mémoire de licence, octobre 2006, Neuchâtel, Université de Neuchâtel, Institut de géographie, 2007, G 15-5747

NATALI, POLI, Città e territori da vivere oggi e domani : il contributo scientifico délie tesi di laurea, Firenze, Firenze University Press, 2007, G 15-5762

NOVELLI, Giovanni, Parco nazionale del Gargano, Roma, Ministero dell'ambiente e della tutela del territorio : Società geografica italiana, 2006, G 15-5740

PELLETIER, Stéphane, L Espagne d'aujourd'hui : politique, économie et société de Franco à Zapatero, Paris, A. Colin, impr. 2008, G 15-5763

PEREDA, Carlos, Inmigraciôn y vivienda enEspaha, Madrid, Ministerio de Trabajo y Asuntos Sociales, DL 2005, G 15-5761

PIGGOTT, Horace Edwin, Great Britain andIreland, London, Dent, 1922, G 15-5770

SALVATORI, Franco, MAUTONE, Parco nazionale del Circeo, Roma, Ministero dell'ambiente e della tutela del territorio : Società geografica italiana, 2006, G 15-5739

SCOTT, James Wesley, EU enlargement, région building and shifting border s of inclusion and exclusion, Aldershot ; Burlington (Vt), Ashgate, cop. 2006, G15-5773

TRISCHITTA, Domenico, Parco nazionale dell'Aspromonte, Roma, Ministero dell'ambiente e della tutela del territorio : Società geografica italiana, 2006, G 15-5745

UNION EUROPÉENNE - POLITIQUE RÉGIONALE, Régions, Actrices du Changement Economique : Réponses de la Politique Régionale aux Défis Démographiques, Luxembourg, Office des Publications Officielles des Communautés Européennes, 2007

URTEAGA, Eguzki, La coopération transfrontalière en pays basque, Paris, Harmattan, 2007, VANDERMOTTEN, Christian, L'identité de l'Europe : histoire et géographie d'une quête d'unité, Paris, A. Colin, DL 2008, G 15-5760

VIVIROLI, Daniel, Ein prozessorientiertes Modellsystem zur Ermittlung seltener Hochwasserabflùsse fur ungemessene Einzugsgebiete der Schweiz : Weiterentwicklung und Anwendung des hydrologischen Modellsystems PREVAH, Bern, Geographisches Institut der Universitàt Bern, cop. 2007, G 15-5757

YVERT-JALU, Anne-Marie Hélène, PERROT, Femmes et famille en Russie d'hier et d'aujourd'hui, Paris, Editions du Sextant, impr. 2007, G 15-5723 


\section{Fonds russe}

GERASFMOV, Morfostruktura i morfoskul ptura platformennyh ravnin SSSR i dna omyvaûsih ego moreï, Moskva, Nauka, 1986, R 1360

KIAN, Everest 82 : Voshozdenie sovetskih al'pinistov na vysocaïsuû versinu Mira, moskva, Fizkul'tura i sport, 1984, R 1417

KONDRAT'EV, K.Â., Geografiâ i kosmos, Moskva, Znanie, 1984, R 1313

Naucnyj simpozium komissiipo ispol'zovaniû zemel'mezdunarodnogo geograficeskogo soûza : tezisy dokladov, [Kiev], Visa Skola, 1976, R 1400

NIKITUK, KAARMA, Sovremennaâ antropologiâ medicine i narodnomu hozâjstvu : Tezisy konferencii, Tallin, oktâbr' 1988g, Tartu, Tartuskij gosudarstvennyj universitet, 1988, R 1412 SLADKOVSKLT, CUMILEV, ZILKIN, FEDOTOV, Zarubeznaâ Aziâ : Vostocnaâ i Central'naâAziâ, Moskva, Mysl', 1982, R 1318

Tartu ùlikooli perioodikavàljaanded 1802-1940 : Registrid, Tartu, Riiklik iilikool, 1983, R 1367<2>

Trakiâ : geografski i istoriceski pregled, Sofiâ, Izdava trakiïskiât naucen institut, 1946, R 1136

USPENSKIÏ, Savva Mihaïlovic, Zivusie vo l'dah, Moskva, Mysl' : 1983, R 1346

VARTAZAROV, Naucnye osnovy racional 'nogo ispol 'zovaniâ, ohrany i upravleniâ vodnymi resursami : 13-âsessiâ 1982 g., [Moskva], izdatel'stvo Moskovskogo universiteta, 1983, R $1390<1$

\section{France}

Améliorer l'accessibilité du parc existant, Paris, Union sociale pour l'habitat, 2005, G 10-5500

Changements climatiques et risques sanitaires en France : [rapport au Premier ministre et au Parlement], Paris, la Documentation française, DL 2007, G 10-5491

CLARIMONT, VLÈS, Tourisme durable en montagne : entre discours et pratiques, Paris, AFNOR, impr. 2007, G 10-5509

DAVEZIES, Laurent, La République et ses territoires : la circulation invisible des richesses, Paris, Seuil, DL 2008, G 10-5489

DAVEZIES, Laurent, La République et ses territoires : la circulation invisible des richesses, Paris, Seuil, DL 2008, G 10-5489

DESVIGNES, MANTEI, MAINBOURG, Améliorer la qualité d'usage touristique des espaces publics en ville : des pistes de réflexion et de sensibilisation pour l'action, Paris, ODIT France, DL 2008, G 10-5552

Développement durable et architecture responsable : engagements et retours d'expériences, [Paris], Ordre des architectes, DL 2007, G 10-5538

Développement économique et emploi : tensions et solidarités au coeur de l'agglomération : éléments de diagnostic pour les travaux de la Conférence métropolitaine, Paris, Atelier parisien d'urbanisme, 2007, G 10-5501

DONZELOT, Jacques, Quand la ville se défait : quelle politique face à la crise des banlieues ?, Paris, Éditions du Seuil, DL 2008, G 10-5485

DRIS, Territoires et territorialité : regards pluridisciplinaires, Paris, l'Harmattan, 2007, G 10-5495 
DUFIEUX, Philippe, GARAIX, DÉLÉAZ, Le rêve de la maison : cités-jardins, lotissements et habitat durable dans le Rhône, Lyon, Conseil d'architecture, d'urbanisme et de l'environnement du Rhône, 2007, G 10-5431

ESTÈBE, Philippe, Gouverner la ville mobile : intercommunalité et démocratie locale, Paris, Presses universitaires de France, impr. 2008, G 10-5505

Formes d'habitat et densités urbaines, [S.1.], Observatoire de la ville, 2007, G 10-5496

\section{G 10-5492}

GODEAU, Eric, MARSEILLE, Le tabac en France de 1940 à nos jours : histoire d'un marché, Paris, Presses de l'Université Paris-Sorbonne, DL 2008, G 10-5547

Guide des projets urbains 2007, Paris, Innovapresse \& Communication, 2007, G 10-5448

HAAS, Sandrine, Les villes moyennes et la santé, Paris, La Documentation française, DL 2008, G 10-5529

Industrie, jeunesse, territoire : cinq thèmes d'une réflexion générale, Nantes, Conseil économique et social des Pays de la Loire, impr. 2007, G 10-5461

JULES SION, Les paysans de la Normandie Orientale, G 13-8

L' architecture de la grande échelle, programme interdisciplinaire de recherche : bilan de l'appel à propositions de recherche : sessions 2006 et 2007, Paris, Ministère de la culture et de la communication : Ministère de l'écologie, du développement et de l'aménagement durables : Ministère du logement et de la ville, impr. 2007, G 10-5498

La gestion des quartiers en chantier, Paris, Union sociale pour l'habitat, 2006, G 10-5499

LACOUR, Claude, THOIN, 40 ans d'aménagement du territoire, Paris, [la Documentation française : DIACT], DL 2008, G 10-5546

LAUBIER, Lucien, La marée noire de l'Erika : quelles conséquences écologiques ?, Paris ; Monaco, Institut océanographique éd., 2007, G 10-5486

LEANDRO, Maria Engracia, Familles portugaises : projets et destins, Paris, CIEMI : Éditions l'Harmattan, DL 1995, G 10-3291

LEFEUVRE, Construire la ville : l'urbanisme en Seine-et-Marne au XXe siècle, Dammarie-lès-Lys, Conseil général de Seine-et-Marne, Direction des archives, du patrimoine et des musées départementaux, DL 2006, G 10-5497

Les adultes du Nord-Pas-de-Calais et la maîtrise des fondamentaux, écrire, communiquer, compter, Lille, INSEE Nord-Pas-de-Calais, 2007, G 10-5502

Les pôles de compétitivité : que peut-on en attendre?, Paris, Ed. Rue d'Ulm, DL 2007, G 10-5510

LESELBAUM, Le maire, l'architecte et l'habitat individuel, [Paris], Ordre des architectes, DL 2007, G 10-5539

MADELINE, MORICEAU, Bâtir dans les campagnes : les enjeux de la construction de la protohistoire au XXIe siècle, Caen, [Maison de la recherche en sciences humaines]: [Presses universitaires de Caen], 2007, G 10-5493

MANTEI, Atlas du tourisme 2007, Paris, ODIT France, DL 2007, G 10-5487

MANTEI, Valorisation touristique du patrimoine et du paysage dans les territoires de montagne : s'évaluer pour évoluer, Paris, ODIT France, DL 2007, G 10-5490 
MERLIN, L 'éco-région d'Ile-de-France : une utopie constructive : réflexion prospective, Paris, la Documentation française, DL 2007, G 10-5545

OBLET, Thierry, Défendre la ville : la police, l'urbanisme et les habitants, Paris, Presses universitaires de France, impr. 2008, G 10-5506

PAILLOT, Christelle, L'arrondissement parisien : entre déconcentration et décentralisation, Paris, L'Harmattan, DL 2007, G 10-5469

Pour un nouvel urbanisme : la ville au cœur du développement durable, Paris ; Gap, Adels : Éd. Yves Michel, DL 2008, G 10-5548

RENARD, Jean, Nantes à la croisée des chemins, Rennes, Presses universitaires de Rennes, 2008, G 10-5549

RENAUD, Philippe, BRENOT, Les retombées radioactives de l'accident de Tchernobyl sur le territoire français : conséquences environnementales et exposition des personnes, Paris, Lavoisier : Editions Tec \& Doc, cop. 2007, G 10-5531

ROBERT, Jean, L'Île-de-France, Paris, Presses universitaires de France, 1994, K 894

ROL-TANGUY, Les déplacements en Ile-de-France : 12 propositions de la DREIF : contribution au débat sur la révision du Schéma directeur de la Région Ile-de-France, Paris, Direction régionale de l'équipement d'Ile-de-France, 2006, G 10-5544

SCoT et développement durable : méthode pour évaluer les schémas de cohérence territoriale au regard des principes du développement durable et définir les indicateurs environnementaux à mettre en place : application au cas de l'agglomération de Montpellier, Lyon, CERTU, impr. 2007, G 10-5488

SCOTde la région d'Annemasse : schéma de cohérence territoriale : des Voirons au Salève, 12 communes pour un projet de territoire, Ambilly, Syndicat d'Études du Genevois Haut-Savoyard, impr. 2007, G 10-5534

TALIANO-DES GARETS, Françoise, ORY, Les métropoles régionales et la culture, 1945-2000, Paris, Comité d'histoire du Ministère de la Culture : la Documentation française, 2007,

VINCENDON, Sibylle, Petit traité des villes à l'usage de ceux qui les habitent, [Paris], Hachette Littératures, impr. 2008, G 10-5542

\section{Géographie Humaine}

ADELKHAH, BAYART, Voyages du développement : émigration, commerce, exil, Paris, Éd. Karthala, impr. 2007, G 9-6642

AINSTEIN, Luis, Modelos de análisis y gestión de redes y componentes urbanos, Buenos Aires, Universidad de Buenos Aires, Facultad de Ciencias Económicas, 2000, G 9-5321

AITCHISON, Cara, KWAN, Geographies o/Muslim Identities : Diaspora, Gender \& Belonging, Ashgate, 2007

ALLAN, Claire, Femmes et politique, Paris, Ellipses, DL 2007, G 9-6604

ALLARD, FOX, PICON, Incertitude et environnement : la fin des certitudes scientifiques, Aix-enProvence, Edisud, impr. 2008, G 9-6679

ANDREOTTI, Giuliana, Paesaggi in movimento : paesaggi in vendita, paesaggi rubati, Trento, V. Trentini, impr. 2007, G 9-6632 
ARCHIVES DÉPARTEMENTALES DU NORD, Traits de Génie: Travaux des Ingénieurs Militaires dans le Nord de la France. Cartes et Plans conservés aux Archives..., Naoned Systems

ASSOCIATION DES INGÉNIEURS TERRITORIAUX DE FRANCE, Ville Durable et ses Territoires de Nature : Politiques Vertes et Evaluations, Dardilly (Rhône), Société des Editions Horticoles de France, 2008

Atlas géostratégique 2008 : une Terre, des hommes, nouvelles frontières, fronts de guerre, Lambesc, Areion, 2007, G 9-6636

BADIE, Atlas de la mondialisation : comprendre l'espace mondial contemporain, Paris, les Presses de Sciences Po, DL 2008, G 9-6680

BASTIÉ, Jean, La ville, Paris ; Milan ; Barcelone, Masson, 1991, G 9-3357

BASTIÉ, Jean, L'espace urbain, Paris ; New York ; Barcelone, Masson, 1980, G 9-2271

BATAILLOU, Christian, Voyages : raisons et pratiques, Perpignan, Presses universitaires de Perpignan, impr. 2007, G 9-6647

BEROUTCHACHVILI, Nikolaï, Geographicalperspectives on sustainable development : a teacher 's guide, Rome ; Moscow ; Beijing, IGU, 2004, G 9-5798

BLANC, Nathalie, Grands Barrages et Habitants : les Risques Sociaux du Développement, Versailles, Quae, 2008

BOISSEAU, Charles Henri (sous la direction), Tourisme Estival de Montagne: Approche comparative des Massifs Français et Etrangers...., ODIT, 2008

BOLOCAN GOLDSTEIN, Matteo, Milano incompiuta : Interpretazioni Urbanistiche del Mutamento, Milano, FrancoAngeli, 2007

BOUQUET, MADELIN, NIVOLLE, Territoires et action sociale, Paris, L'Harmattan, DL 2007, G 9-6640

Cadastrale : le Cadastre a 200 ans, Conseil Général de la Meuse, 2007

CARRARO, Carlo, Climate and Trade Policy, Elgar Publishing, 2007

CARREIRO, SONG, WU, Ecology, planning, and management of urban forests : international perspectives, New York, Springer, cop. 2008, G 9-6630

CHAMPEYRACHE, Clotilde, Sociétés du crime : un tour du monde des mafias, Paris, CNRS éd., impr. 2007, G 9-6644

CHATELUT, Jean, Chronique d'un Vivant Village : du Nécessaire aux indispensables Initiatives, PaYse Editions, 2008

CHIARENTI, Francesco, Riflessioni e Osservazioni sull 'Agricoltura Toscana e Particolarmente sull Istituzione de ' fattori, Firenze, Polistampa, 2007

CHICK, Martin, Electricity and energy policy in Britain, France and the United States since 1945, Cheltenham ; Northampton, Mass., E. Elgar, cop. 2007, G 9-6690

COMITÉ DES GRANS PRrX NATIONAUX DE L'ARCHITECTURE, Faut-il protéger les Grands Ensembles ?, Ministère de la Culture et de la Communication/ Drection de l'Architecture et du Patrimoine, 2007

DAGRADI, Piero, Geografia della popolazione, Bologna, Pàtron ed., 2006, G 9-6651

DAVIDSON, Joyce, Emotional Geographies, Ashgate, 2007

DEZERT, Bernard, La Crise mondiale de l'énergie : ses implications géographiques, Paris, Éditions SEDES-C.D.U. réunis, 1981, G 9-2383 
ERMINI, Leonardo, Linee Guida Gestionali per gli Ambienti Naturali e Semi-Naturali Lungo il Corso dell'Arno, Milano, FrancoAngeli, 2007

Estudios turisticos, Madrid, Ministerio de Comercio y Turismo. Instituto Espan, ol de Turismo

FAVAREL, Françoise, Plan Local d'Urbanisme : 10 Thèmes Clés pour débattre du Projet urbain, Commission Urbanisme de l'Ordre des Architectes, 2007

FEARNE, Andrew, VOERLEY, Regoverning Markets : a Place for Small-Scale Producers in Modem Agrifood Chains?, Ashgate, 2007

FIJALKOW, Yankel, Sociologie des villes, Paris, la Découverte, impr. 2007, G 9-6649

Finance et délocalisations, Paris, Association d'économie financière, DL 2007, G 9-6681

FURFARI, Samuele, Le monde et l'énergie, enjeux géopolitiques, Paris, Éd. Technip, 2007,

G 9-6633

GASPAR, LUCIA, O Recife : Uma Bibliografia, Recife (Fundaçao Joaquim Nabuco), Massangana, 2008

GOTTMANN, Jean, La politique des Etats et leur géographie, Paris, A. Colin, 1952, G 9-584

GOTTMANN, Jean, La politique des États et leur géographie, Paris, A. Colin, 1952, G 9-584

GOUJON, Philippe, DUQUENOY, Information Society: Innovation, legitimacy, Ethics and Democracy, Springer, 2007

GOUNOT, JALLAT, CARITEY, DEFRANCE, Les politiques au stade : étude comparée des manifestations sportives du XIXe au XXIe siècle, Rennes, Presses universitaires de Rennes, 2007, G 9-6646

GROUPEMENT DE RECHERCHE SUR LES INSTITUTIONS ET LE DROIT DE L'MÉNAGEMENT, DE

L'URBANISME ET DE L'HABITAT (FRANCE) -Droit de l'aménagement, de l'urbanisme, de l'habitat : 2003, Paris, le Moniteur, 2003, G 9-5608

GUILLAUD, Yann, Biodiversité et développement durable, Paris, Unesco : Karthala, impr. 2007, G 9-6641

HAGGETT, Peter, FRECHOU, L'analyse spatiale en géographie humaine, Paris, A. Colin, impr. 1973, G 9-1623

HARVEY, David, Géographie de la Domination, Les Prairies Ordinaires, 2008

HARVEY, David, Spaces of Hope, University of California Press, 2000

HOERNER, Jean-Michel, Géopolitique du tourisme, Paris, A. Colin, impr. 2008, G 9-6656

HÔHN, Charlotte, People Population Change andPolicies : l.Lessonsfrom the Population Policy Acceptance Study : 2. Démographie Knowledge - Gender - Ageing, Springer, 2008

JACQUES LOURD, Le lin et l'industrie linière, K 346

JAPHET KEYOU, Mathias, La performance économique des pays en développement : constats et défis : étude comparative entre les pays de l'Afrique subsaharienne et de l'Asie de l'Est et du Sud-Est, Paris, Publibook, cop. 2007, G 9-6634

KALAORA, Bernard, Les cités du littoral. Le littoral dans tous ses états, [SA.], [s.n.], G 9-4743-<p. 76-88>

KOCSIS, Karoly, SCHWEITZER, Ukraine inMaps, Geographical Research Institute Hungarian Academy of Sciences, 2008 
KOUL, Johann Georg, Der Verkehr und die Ansiedelungen der Menschen in ihrer Abhàngigkeit von der Gestaltung der Erdoberflàche, Dresden und Leipzig, Arnold, 1841, G 9-385

LECOQUIERRE, Bruno, Parcourir la Terre : le voyage, de l'exploration au tourisme, Paris, l'Harmattan, DL 2008, G 9-6677

LEROND, Michel, Le développement soutenable : évaluation simplifiée dans un contexte Nord-Sud, Paris, L'Harmattan, DL 2007, G 9-6643

LEVAYE, Patrick, Géopolitique du catholicisme, Paris, Ellipses, DL 2007, G 9-6674

LÉVY, L'invention du monde : une géographie de la mondialisation, Paris, Sciences Po, les presses, impr. 2008, G 9-6682

LYNCH, Kevin, VÉNARD, VÉNARD, L'image de la cité, Paris, Dunod, 1969, G 9-1520

MANCHESTER, Ernest A., Collins 'pocket atlas ofthe world : by Ernest A. Manchester, ..., London ; Glasgow, Collins' clear-type press, [19??], G 9-6686

MANSFELD, Yoel, Christian Tourism to the Holy Land: Pilgrimage during Security Crisis, Ashgate, 2006

Menaces contre la planète : bouleversements climatiques, hivers nucléaires, orages solaires... Vérités ou imposture?, Lambesc, Areion, 2007, G 9-6637

MICHALET, BOISSIEU, BOURLÈS, CHEVALIER, Le défi du développement indépendant, Paris, Les éd. Rochevignes, DL 1983, G 9-6696

MISTRY, BERARDI, Savonnas and dry fore sts : linking people with nature, Aldershot (England) ; Burlington (Vt), Ashgate, cop. 2006, G 9-6692

MOINE, Alexandre, Le territoire : comment observer un système complexe, Paris, L'Harmattan, DL 2007, G 9-6592

MOKASS, Mustapha, BARBUT, Comment amorcer le marché mondial de l'énergie propre : nouvelles énergies et climat : un destin lié, Paris, l'Harmattan, DL 2007, G 9-6645

MONNOYER-LONGÉ, TERNAUX, Mondialisation des services : innovation et dynamiques territoriales, Paris, l'Harmattan, DL 2007, G 9-6638

MONTEL-DUMONT, Développement et environnement, Paris, la Documentation française, 2007, G 9-6684

MONTGOMERY, John R., The new wealth of cities : city dynamics and thefifih wave, Aldershot ; Burlington (Vt.), Ashgate, cop. 2007, G 9-6695

MOSCA, ROBERT A, Projezione Internazionale delT Economia Pugliese, Soveria Mannelli (Cantanzaro, Rubbettino, 2007

MÜLLER-WIELAND, Birgit, La bataille des OGM : combat vital ou d'arrière-garde ?, Paris, Ellipses, impr. 2007, G 9-6631

OBAID, State of world population : 2003 : making 1 billion count : investing in adolescents' health and rights, New York, UNFPA, cop. 2003, G 9-5661

OECD historical statistics, 1970-1999, Paris, Organisation for Economic Co-operation and Development, 2001, G 9-5011

PASKOFF, Roland, L'érosion des plages : les causes, les remèdes, Paris ; Monaco, Institut océanographique, DL 2007, G 9-6639

PÉBEREAU, Économie politique contemporaine, Paris, A. Colin, impr. 2007, G 9-6655 
Pétrole et gaz : quels maîtres du Monde?, Lambesc, Areion, 2007, G 9-6635

PEZEU-MASSABUAU, Jacques, Les demeures de la solitude .formes et lieux de notre isolement, Paris, l'Harmattan, DL 2007, G 9-6685

PONTI, Marco, ERBA, Una Politica per i Trasporti Italiani, Roma, GLF editori Laterza,

RATZEL, Friedrich, Anthropo-Géographie oder Grundzùnge der Anwendung der Erdkunde ouf die Geschichte, Stuttgart, J. Engelhorn, 1882, G 9-8

RATZEL, Friedrich, Anthropogeographie..., Stuttgart, J. Engelhorn, 1891, G 9-9

ROBIN, Marie-Monique, HULOT, Le monde selon Monsanto : de la dioxine aux OGM, une multinationale qui vous veut du bien, Paris, Ed. la Découverte : Arte éd., DL 2008, G 9-6683

SACCHI, Livio, Rappresentazione délia Citta_ : Pescara e il Sistema Territoriale : Piano di Monitoraggio e Riqualificazione dei Bacini Fluviali..., Roma, Officina, 2007

SMITH, Heather, Latinos in the New South : Transformations of place, Ashgate, 2006

THOMAS, Isabelle, Transportation networks and the optimal location of human activities : a numerical geography approach, Cheltenham, UK, E. Elgar, 2002, G 9-6689

Utilisation du sol et diversité biologique dans les Alpes : Faits, perspectives, recommandations, Zurich (Suisse), VDF-Hochschulverlag AG, 2007

VEYRET-MEKDJIAN, Le développement durable, Paris, Éd. Sedes, impr. 2007, G 9-6602

WACKERMANN, Gabriel, Géographie des Civilisations, Ellipses, 2008

WESCOAT, JOHNSTON, Political economies of landscape change : places of integrative power, Dordrecht ; London, Springer, cop. 2008, G 9-6629

ZETTER, R, Designing sustainable Cities in the Developing World, Ashgate, 2006

\section{Géographie Physique}

BARNAUD, Geneviève, Conserver les milieux humides : pourquoi ? comment ?, Versailles ; Dijon, Quae : Educagri éditions, DL 2007, G 8-3195

CARPINE-LANCRE, The history of GEBCO 1903-2003 : the 100-year story of the General Bathymetric Chart of the Oceans, Lemmer, The Netherlands, GITC bv, c2003, G 8-2924

DEMANGEOT, Jean, Les milieux « naturels » du globe, Paris ; Milan ; Barcelone, Masson, 1990, G 8-2141

DEWOLF, BOURRIÉ, FREYTET, JOLY, LETERRIER, Les formations superficielles : genèse, typologie, classification, paysages et environnements, ressources et risques, Paris, Ellipses, impr. 2007,

G 8-3197

FLOHN, LANDSBERG, General climatology, Amsterdam ; London ; New York, Elsevier, 1969, G 8-1339-2

GUPTA, Large rivers : geomorphology and management, Chichester, J. Wiley, cop. 2007, G 8-3196

LANG, LAVABRE, SAUQUET, RENARD, Estimation de la crue centennale pour les plans de prévention des risques d'inondations, Versailles, Editions Quae, 2007, G 8-3194

Lavoisier, cop. 2008, G 8-3199 
MARTONNE, Emmanuel de, CHEVALIER, CUÉNOT, Traité de géographie physique, Paris, A. Colin,

1932, G 8-392-<3>

PERRET, FAROLFI, HASSAN, Water governance for sustainable development, London ; Sterling (Va) ; Montpellier, Earthscan : Cirad Editions, cop. 2006, G8-3205CARREGA, Information géographique et climatologie, Paris, Hermes Science publication :

\section{Monde arabe-Proche-Orient}

FEKI, Masri, Israël : géopolitique et enjeux, Levallois-Perret, Studyrama, impr. 2008, G 24-396

MUTIN, Georges, BARGE, Le Moyen-Orient : peuples et territoires, Paris, Ellipses, DL 2007, G 24-398

Revue annuelle des Émirats Arabes Unis 2006, London, Trident Press, cop. 2006, G 24-393

TESSA, Ahmed, Algérie : histoire d'une construction spatiale : 1960-2005, Paris, Publisud, DL 2007, G 24-397

TYNER, James A., The business ofwar : workers, warriors andhostages in occupiedIraq, Aldershot ; Burlington, Vt, Ashgate, cop. 2006, G 24-403

VERDEIL, FAOUR, VELUT, Atlas du Liban : territoires et société, Beyrouth, Institut français du Proche-Orient : Conseil national de la recherche scientifique, DL 2007, G 24-395

\section{Périodiques}

3 regards sur l'Union européenne, Bruxelles, Fédération des Professeurs de Géographie, 2007, P 1704-62

ABDALLAH, Vie associative, action citoyenne, [S.l.], [s.n.], P 4530-1229

Agreste, Paris, Ministère de l'agriculture et de la pêche, SCEES, 2000-, P 4430-194

Agreste, Paris, SCEES, 1996-, P 1647-1

Anales de geografía de la Universidad Complutense, Madrid, Universidad Complutense de Madrid, Servicio de Publicaciones, P 3943-27-1

Annals of tourism research, New York, NY, Pergamon, 19??-, P 4640

Arctic, antarctic, and alpine research, Boulder, CO, Institute of Arctic and Alpine Research, University of Colorado at Boulder, P 4009

Atlantic geology, Wolfville, NS, The Society, P 3022-43/1

Au fil du Rhin, Strasbourg, Ed. de la Navigation du Rhin, 2006, P 2112-\%08-03

AUDOUIN, Traits urbains : le mensuel opérationnel des acteurs du développement et du renouvellement urbains, Paris, Agence Innovapresse, 2005-, P 4725

BANZO, PATO E SILVA, VALETTE, Lisbonne en ses périphéries, Toulouse, Presses universitaires du Mirail, DL 2007, P 460/A-24

BASTIN, Stéphane, DECROLY, Changes in west african territories, [Bruxelles], Société royale belge de géographie : Société belge d'études géographiques, 2007, P 4599-4

BAUMANN, Eveline, Risques et microfinance, Paris ; Bondy, A. Colin : Institut de recherche pour le développement, DL 2007, P 1652/A-44 
BECK, BENARROUS, DEREX, Les zones humides européennes : espaces productifs d'hier et d'aujourd'hui, Cordemais, Estuarium, 2007, P 4613-9

BELGEO : revue belge de géographie : = Belgisch tijdschrift voor geografie : = Belgische Zeitschrift für Geographie : = Belgian Journal of Geography, Leuven, KUL, P 4599

Berichte zur Deutschen Landeskunde, [s.l.], Zentralausschuss für Deutsche Landeskunde e. V, 1942-, P 1014

BEYLICH, Achim A., SCHMIDT, Sedimentary source-to-sink-fluxes and sédiment budgets in changing cold environments, Berlin, Gebriider Borntraeger, 2008, P 10-52-NS-1

BLÜMEL, BORCHERDT, HAHN, Stuttgarter geographische Studien, Stuttgart, Geographisches Institut der Universität Stuttgart, 1924-, P 1332

Boletim de ciências geodésicas, Curitiba, Imprensa Universitária, P 4554-13/1

BONNIER, Le Mont Ventoux, Le Tholonet, Forêt méditerranéenne, 2007, P 4668-28-4

BOST, Les zones franches, Paris, A. Colin, 2007, P 523/A-658

Bulletin climatique mensuel, Boulogne-Billancourt, Direction de la météorologie nationale, Service central d'exploitation de la météorologie, 1989-, P 4217

Bulletin de la Société de géographie d'Egypte, Le Caire, Geographical Society of Egypt, 1876-, P 937-80

Bulletin de la Société géologique de France, Paris, Société géologique de France, 1830-, P 454

Bulletin des bibliothèques de France, Paris, Direction des bibliothèques de France, 1956-, P 1267

Bulletin of Latin American research, Oxford ; Oxford ; Oxford, Oxford Microform Publications :

Oxford Microform Publications : Blackwell, 1981-, P 3978

Bulletin, Alger, Office national de la géologie, 1986-1991, P 844

BURGEL, NDONGMBA, Villes du Gabon, Nanterre, Laboratoire de géographie urbaine de

l'Université Paris X-Nanterre, 2007, P 3695-40/41

Cahiers des Amériques Latines, Paris, Université de la Sorbonne nouvelle Paris 3, Institut des hautes études d'Amérique latine, 1985-, P 3074-51/52

CAMBREZY, CHARBIT, Migrations internationales et vulnérabilités, Poitiers, Association pour l'étude des migra 